Data fusion of ultrasound and GPR signals for analysis of historic walls

This article has been downloaded from IOPscience. Please scroll down to see the full text article.

2012 IOP Conf. Ser.: Mater. Sci. Eng. 42012008

(http://iopscience.iop.org/1757-899X/42/1/012008)

View the table of contents for this issue, or go to the journal homepage for more

Download details:

IP Address: 158.42.243.38

The article was downloaded on 22/03/2013 at 08:57

Please note that terms and conditions apply. 


\title{
Data fusion of ultrasound and GPR signals for analysis of historic walls
}

\author{
A Salazar, J Gosalbez, G Safont and L Vergara \\ Universitat Politècnica de Valencia, Institute of Telecommunications and Multimedia \\ Applications, Camino de Vera s/n, 46022, Valencia, Spain \\ E-mail: asalazar@dcom.upv.es
}

\begin{abstract}
This paper presents an application of ultrasounds and ground-penetrating radar (GPR) for analysis of historic walls. The objectives are to characterize the deformation of a historic wall under different levels of load weights and to obtain an enhanced image of the wall. A new method that fuses data from ultrasound and GPR traces is proposed which is based on order statistics digital filters. Application results are presented for non destructive testing (NDT) of two replicates of historic ashlars' masonry walls: the first one homogeneous and the second one containing controlled defects such as cracks and nooks. The walls are measured separately using ultrasounds and GPR at different load steps. Time and frequency parameters extracted from the signals and different B-Scans for each of the NDT techniques are obtained. After this, a new fused representation is obtained, which results demonstrate the improvement of characterization and defect detection in historic walls using data fusion.
\end{abstract}

\section{Introduction}

The principal objective of this paper is to provide an approach for non-destructive testing (NDT) based on ultrasound and ground-penetrating radar (GPR) signal processing for diagnoses of historical walls. Common materials employed in heritage buildings are stone, mortar and brick. This kind of constructions resists well the pass of the time; nonetheless, degradation processes are inevitable and difficulties arise for measuring its degree of degradation. The degradation processes affect both structural (cracks, fissures, detachments, displacements...) and aesthetic (dirt, crusts, efflorescence...) characteristics of the historical buildings. Among the main pathologies that cause building breakdown are humidity damages caused by capillarity ascent, breeze or high humidity environments, successive freeze-thaw cycles that result in crystallization, and broken mortar joints. At present, to deal with structural evaluation, it is usual to use destructive testing by means of the extraction and characterization of tubes from the material that are characterized using classical morphological and physiochemical analyses. Thus, considering heritage value preservation, NDT is being increasingly applied in pathological and structural diagnoses of historical buildings.

In this paper, we present results of testing two scale models of historic ashlar masonry walls using through-transmission ultrasounds (two transducers, an emitter and a receiver each one located at opposite sides of the wall) and pulse-echo GPR (an antenna used as emitter and receiver located at one side of the wall). The walls were built with travertine ashlars from Godella's quarry in Spain and the dimension of the ashlars was $40 \times 30 \times 20 \mathrm{~cm}$ (see figure 1). The final dimension of the two walls was $287 \mathrm{~cm}$ length, $220 \mathrm{~cm}$ height and $20 \mathrm{~cm}$ thickness. Impoverished mortar with a low compression resistance $(<4 \mathrm{MPa})$ was used as binding material. One of the walls was homogeneous and the second 
one was mechanized including artificial defects (two drill holes, a vertical flaw and a crack or nook filled with mortar, see figure 2). The walls were divided in 7 rows and 7 columns as shown in figure 2 . A total of 42 ultrasound measurements were taken in the positions indicated by blue crosses and the radar signals were collected using a survey cart with encoder through the trajectories depicted by red dash-dot lines in figure 2. In order to evaluate the sensitivity of the NDT methods to tensional states of the walls, they were introduced in a hydraulic press and loaded with 10Tn, 50Tn and 80Tn.

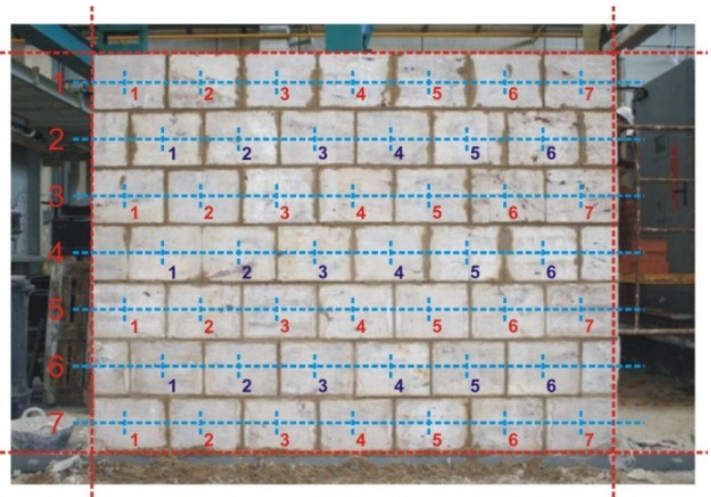

Figure 1. Ashlar inhomogeneous masonry wall.

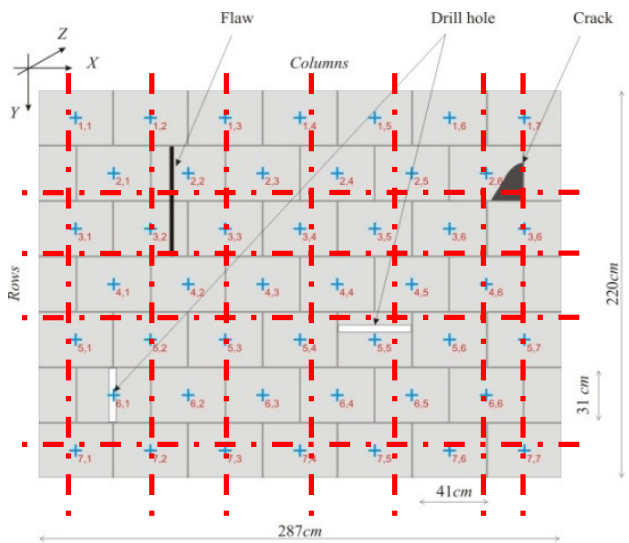

Figure 2. Defect and measurement outline.

Ultrasonic and GPR inspection are based on mechanical and electromagnetic waves, respectively. These waves propagate inside the material structure and its response is measured by signals that contain the reflections produced by the material grain microstructure plus the echoes caused by the inhomogeneities inside the material [1][2]. Signal processing allows extraction of information for characterization of the propagation medium and for detecting material in-homogeneities [3]. In this paper, we present results in detection of detects in the wall scale models by ultrasounds and GPR separately (Section 2 and 3) and enhanced results (Section 4) obtained by fusing both results. Data fusion is a new method that is progressively finding more applications in NDT, such as fusion of Xray and ultrasound images in welds; and infrared thermography imagery in nuclear applications. The fusion methods employed have been based on classification systems and recently data fusion was done using possibility theory and fuzzy sets [4]. The fusion method presented here combines ultrasound and radar images estimated from the propagation velocity and signal power, respectively. From the original measurements, 2D images are formed using interpolation and morphological transformations, and after fusion, the fused image is filtered using an order statistical digital filter.

\section{Ultrasonic tomography}

An ultrasonic signal (A-Scan) was measured for each spatial position described in figure 2. The following parameters were extracted from ultrasonic signals: propagation velocity (V), signal power (P) and maximum frequency (fmax), see table 1. The temporal window used for their estimation

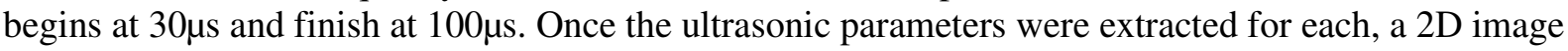
usually called B-Scan can be obtained. In this ultrasonic image or tomography $\mathrm{X}$ axis is associated to the length and $\mathrm{Y}$ axis is associated to height of the wall. The color of the image represents the value of the ultrasonic parameter. The variation of the color represents the variation of the ultrasonic parameters that can be related to the shape and spatial position of the defects. In order to obtain realistic visualization (smoothed eliminating sharp edges) of the wall tomography, a classical linear interpolation algorithm called ordinary Kriging was applied [5].

Several tomographies were estimated for each of the ultrasonic parameters and load steps. The variations of the tomography values that were expecting to associate with defects, were masked by variations of the own material and measurement variance. Thus, differential tomographies were obtained between subtracting tomographies corresponding to different load steps (e.g., subtracting 
10Tn tomography from 50Tn tomography). By this way, the variations between the ultrasonic parameter and the variations of the load were compared (ultrasonic parameters are sensitive to tensional state; velocity and signal power suffer an increment with load whereas central frequency is kept constant). Figure 3 shows a differential 50Tn-10Tn tomography, wall outline over imposed, where some defects are detected. The rationale is that ultrasonic dependence with load is different between sound and unsound zones and ultrasonic parameters suffer different percentage of variation, as reflected in figure 3. The vertical flaw and horizontal drill hole were not detected because the load was not so high to suppose an important variation of the ultrasonic parameter, in this case, velocity. The crack is detected and it is possible to note how this defect affects adjacent ashlars.

Table 1. Parameters extracted from the ultrasonic signals.

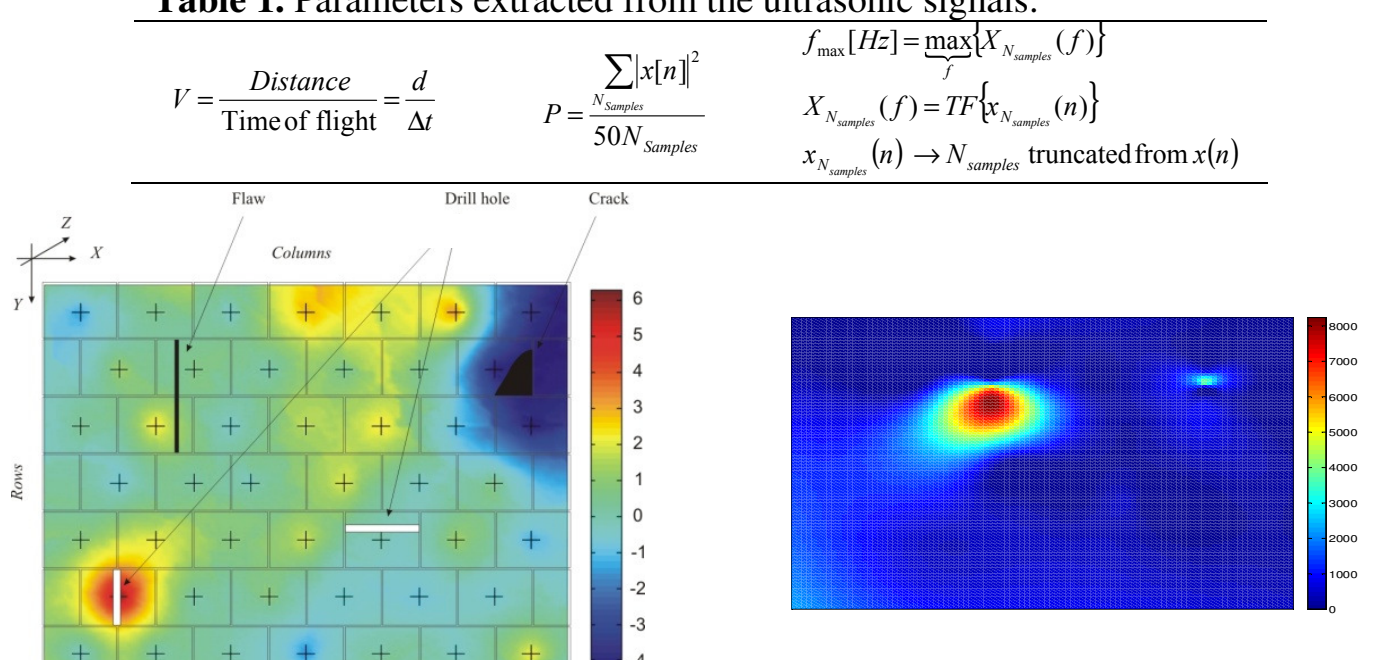

Figure 3. Ultrasound tomography.

Figure 4.GPR tomography.

\section{GPR tomography}

Processing of GPR signals included three common processing steps: background signal removal, depth resolution enhancing and Kirchoff migration. The result of this processing is a radargram, which is an image (also called B-Scan) that represents values of the measured signal (in this case we used signal power) at different depths of the material through the points of a trajectory (defined in figure 2). The objective of the several processing steps was to highlight the zones in the radargram where the inhomogeneities were located. Thus, envelope estimation and automatic gain control were also implemented for data interpretation and to enhance the image contrast. A tomography was obtained by merging the 11 radargrams obtained for each of the GPR trajectories. This image was cleaned applying morphological operations using disk-shaped structuring element with a radius of 5 pixels and thresholding power values to .7 of the maximum. This last guarantees only high reflections to be represented and filtering spurious reflections. After this, as in the ultrasound tomography processing, an interpolation algorithm was used to generate a smoothed tomography. Figure 4 shows the GPR tomography obtained for the inhomogeneous wall at 50Tn load step. The high reflection produced by the flaw and the power decrease caused by the crack are depicted. These defects affect wave propagation travelling depending on its shape in two different ways, in the case of the crack; the propagated waves have to surround the defects and therefore their energy decreases. In the case of the flaw, multiple reflections and diffraction with the defect borders arise.

\section{Results and Conclusion}

Figures 5 and 6 represent fused tomographies obtained from the ultrasound and GPR images explained in Section 2 and 3. The operators for combining the images were product and sample mean for figures 5 and 6, respectively. After fusion, the fused image was filtered using a median filter. Figure 6 shows 
an enhanced tomography were three of the four defects (flaw, crack and vertical drill) are shown and spurious defective areas seem more attenuated.

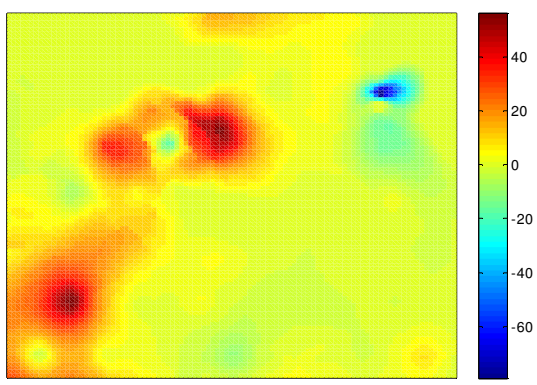

Figure 5. Fused tomography by product.

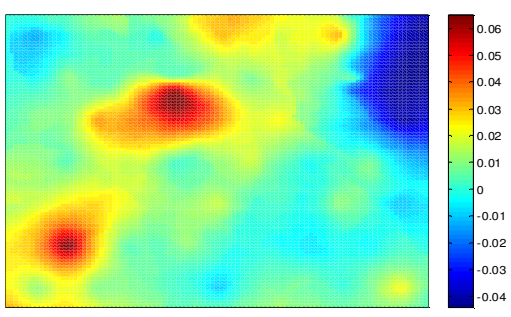

Figure 6. Fused tomography by sample mean.

We observe that the different fusion methods produce different results. An explanation for that may be done in the framework of optimal estimation theory. Thus we may consider that the final goal of the problem is to estimate a parameter, associated to every point in the image, represented by the amplitude value of every pixel. This parameter is supposed to change (normally increasing) due to the presence of a defect. The two values respectively measured by GPR and ultrasonic methods may be considered "observations" from which the parameter is to be estimated. In Bayes estimation theory [6], the parameter and the observations are considered random variables having some marginal and joint distributions, and the optimum estimator corresponds to the one minimizing some cost function. It is, for example, well-known, that the minimum mean-square error (MMSE) estimator is given by the expected value of the parameter conditioned to the observations. In general, this conditioned expected value is a nonlinear function of the observations, but if the marginal and joint distributions of the parameters and the observations are Gaussian the optimum estimator of the parameter is a linear combination of the observations. Actually, if the observations are uncorrelated and have equal crosscorrelation to the parameter, the optimum estimator is just the sample mean of the observation, which is one the fusion method that we have implemented.

Following a similar interpretation, the fusion implementing the product is optimum when the marginal and joint distributions of the parameter and the observation are log-normal (equivalent to a linear combination after taking logarithms). In a general case the appropriate non-linear function will depend on the underlying distributions. Among them those ones based in order statistics [7] are very popular. Thus, we have experimented also with the fusion based in selecting the maximum value of the two observations, although other methods could be tried. Considering the obtained results it seems that the Gaussianity and uncorrelation assumptions are reasonable in our case, as the best performance has been obtained with the fusion implementing the sample mean of the two images.

\section{Acknowledgments}

This work has been supported by Generalitat Valenciana under grant PROMETEO/2010/040, and Spanish Administration and European Union FEDER Prog. under grant TEC2011-23403 01/01/2012.

\section{References}

[1] Cheeke J D 2002 Fundamentals and Applications of Ultrasonic Waves (Florida: CRC Press)

[2] Salazar A and Vergara L 2010 Eurasip Journal on Advances in Signal Processing 2010125201

[3] Salazar A Vergara L and Llinares R 2010 Mechan. Systems and Signal Proc. 24 (6) 1870-1886

[4] Ploix M-A, Garnier V, Breysse D, and Moysan J 2011 NDT\&E International 44 442-448

[5] Journel A G and Huijbregts Ch J 2004 Mining Geostatistics (New Jersey :The Blackburn Press)

[6] Kay S M 1993 Fund. of Statistical Signal Processing, Estimation Theory vol 1 (New Jersey: Prentice Hall)

[7] Pitas I and Venetsanopoulos AN 1990 Nonlinear Digital Filters (Boston:Kluwer Academic) 